\title{
Estoque de carbono e atributos físicos de um Latossolo em cronossequência sob diferentes manejos ${ }^{1}$
}

\author{
Karina de V. Rossetti ${ }^{2}$ \& José F. Centurion ${ }^{3}$
}

\author{
Palavras-chave: \\ matéria orgânica do solo \\ plantio direto \\ sistema convencional
}

\begin{abstract}
R E S U M O
Propôs, neste trabalho, determinar o efeito do sistema plantio direto (SPD) sob diferentes anos de implantação e do preparo convencional (SPC) cultivados por dois anos agrícolas, com soja e milho, sobre os estoques de carbono e atributos físicos de um Latossolo Vermelho em comparação com a mata nativa. O delineamento foi inteiramente casualizado, em parcelas subdivididas (cinco tratamentos e três camadas), com quatro repetições. Os tratamentos em 2008/09 foram: SPD com 5 (SPD5), SPD com 7 (SPD7), SPD com 9 (SPD9) anos de implantação, SPC e uma área de mata nativa $(\mathrm{MN})$. Os tratamentos se estenderam para o ano de 2009/10, assim identificados: SPD6, SPD8, SPD10 e SPC e MN; as camadas de solo estudadas foram: 0-10, 10-20 e 20-30 cm. Os resultados obtidos mostraram que a maioria dos tratamentos manteve o teor do estoque de carbono (EC) durante a transição da camada superficial para a subsuperficial. O maior EC foi mais pronunciado na camada superficial na qual os SPD9 e SPD10 foram os mais relevantes em comparação com os demais sistemas conservacionistas.
\end{abstract}

\section{Key words:}

soil organic matter

no-tillage

conventional tillage

\section{Carbon stock and physical attributes of an Oxisol in chronosequence under different managements practices}

\begin{abstract}
A B S T R A C T
The aim of this study was to determine the effect of the no-tillage planting systems (NT) under different years of implantation and of conventional tillage, growing soybeans and maize for two crop years, on carbon stocks and physical attributes of a Red Oxisol in comparison to native forest. A completely randomized design with split-plots (five treatments and three layers) was used, with four replications. The treatments in 2008/09 were: NT with 5 (NT5); NT with 7 (NT7); NT with 9 (NT9) years of implantation, conventional tillage (CT) and a native forest area (NF). The treatments extended to the year 2009/10, identified as: NT6, NT8, NT10 and CT and NF; the studied soil layers were: 0-10, 10-20 and $20-30 \mathrm{~cm}$. The results obtained showed that most of the treatments maintained the carbon stock content (CS) during transition from the surface layer to the underground layer. CS was more pronounced in the surface layer and, within it, the NT9 and NT10 were the most relevant in comparison to the other conservationist systems.
\end{abstract}

Protocolo 036-2014 - 01/02/2014 • Aprovado em 07/11/2014 • Publicado em 26/01/2015

${ }^{1}$ Parte da Dissertação de Mestrado da primeira autora junto ao Programa de Pós-Graduação em Agronomia (Ciência do Solo) da UNESP

${ }^{2}$ Doutoranda em Agronomia (Produção Vegetal)/UNESP. Jaboticabal, SP. E-mail: krossetti@bol.com.br (Autora correspondente). Bolsista Capes

${ }^{3}$ UNESP. Jaboticabal, SP. E-mail: jfcentur@fcav.unesp.br. Bolsista CNPq 


\section{INTRODUÇÃO}

O solo mantido sob vegetação nativa oferece condições adequadas ao desenvolvimento das plantas e quando há transformação desses ambientes em agricultura convencional também ocorrem, em geral, mudanças drásticas na qualidade física do solo em perda de teores de matéria orgânica (Vezzani \& Mielniczuk, 2011). Neste aspecto, a adoção de sistemas de manejo do solo considerados conservacionistas, tais como o sistema plantio direto (SPD) tem-se apresentado como alternativa viável contra a perda de matéria orgânica contribuindo para assegurar a sustentabilidade do uso agrícola do solo. A viabilidade do SPD decorre da manutenção dos resíduos culturais na superfície do solo associados à rotação de culturas anuais que propicia decomposição gradual e posterior acúmulo de material orgânico no perfil do solo (Franzluebbers et al., 2007).

Comumente, os solos apresentam, no plantio direto e após três a quatro anos de cultivo, valores mais altos de densidade do solo e microporosidade na camada superficial, quando comparados com outros sistemas de manejo (Stone \& Silveira, 2001). Tal padrão decorre do arranjamento natural do solo quando não é mobilizado e da pressão promovida pelo trânsito de máquinas e implementos agrícolas, sobremaneira quando realizado em solos argilosos e com teores de umidade (Tormena et al., 1998). Entretanto, no decorrer do tempo a densidade do solo sob SPD tende a diminuir devido, em parte, ao aumento do teor de matéria orgânica na camada superficial melhorando a agregação e aumentando a porosidade total do solo (Tormena et al., 1998).

Além dos benefícios do aumento do teor de matéria orgânica do solo sobre atributos físicos, o aumento no estoque de $\mathrm{C}$ no solo resulta na retirada de $\mathrm{CO}_{2}$ da atmosfera contribuindo para a mitigação do efeito estufa (Cerri et al., 2010). Este padrão está associado ao aporte de resíduos vegetais e à ausência da mobilização excessiva do solo via seu preparo, o qual diminui a exposição do $\mathrm{C}$ protegido nos agregados ao ataque da comunidade microbiana retardando o processo de decomposição (Al-Kaisi \& Yin, 2005). Assim, a quantificação do estoque de carbono no solo e a avaliação de seu grau de estabilidade são, portanto, medidas importantes no processo de identificação de práticas agrícolas mais adequadas com o intuito de sequestrar carbono da atmosfera.

O objetivo deste trabalho foi quantificar os estoques de carbono orgânico e atributos físicos de um Latossolo em uma cronossequência sob diferentes manejos.

\section{Material e Métodos}

O experimento foi realizado em Jaboticabal, SP, situado nas coordenadas geográficas de $21^{\circ} 14^{\prime} 05^{\prime \prime}$ de latitude Sul, $48^{\circ} 17^{\prime}$ 09" de longitude Oeste, com altitude média de 615,01 m. O clima da região, de acordo com a classificação climática de Köppen, é do tipo Cwa, com verão quente e inverno seco, precipitação pluvial média anual de $1.428 \mathrm{~mm}$ e temperatura média de $21^{\circ} \mathrm{C}$. O solo da área de estudo foi classificado como Latossolo Vermelho distrófico típico, textura média, A moderado, caulinítico, hipoférrico, muito profundo e com relevo plano a suave ondulado (EMBRAPA, 2013), com $330 \mathrm{~g} \mathrm{~kg}^{-1}$ de argila, $40 \mathrm{~g} \mathrm{~kg}^{-1}$ de silte e $630 \mathrm{~g} \mathrm{~kg}^{-1}$ de areia na camada de 0-30 cm (EMBRAPA, 2011).

Antes da implantação do experimento o solo vinha sendo cultivado em sistema de preparo convencional durante 30 anos, com sucessão anual de milho e soja e safrinha de milho, no outono. A partir dos anos 2000, 2002 e 2004, foram implantados, respectivamente para o ano agrícola de 2008/09 o SPD com 9 (SPD9), o SPD com 7 (SPD7) e o SPD com 5 (SPD5) e para o ano agrícola de 2009/10, respectivamente, o SPD com 10 (SPD10), o SPD com 8 (SPD8) e o SPD com 6 (SPD6) anos de implantação. No ano agrícola 2008/09 quatro partes da área, com $60 \mathrm{~m}^{2}$ foram destinadas ao sistema de preparo convencional (SPC); as demais áreas dos sistemas de manejo foram demarcadas também com $60 \mathrm{~m}^{2}$ cada qual. $\mathrm{O}$ SPC foi constituído de uma escarificação do solo até $30 \mathrm{~cm}$ de profundidade seguida de uma gradagem pesada e uma gradagem leve.

Em novembro de 2008 foi realizada a semeadura da soja, cultivar M-SOY 6101 em profundidade de $3 \mathrm{~cm}$ e espaçamento de $45 \mathrm{~cm}$ entrelinhas e a adubação na semeadura consistiu de $250 \mathrm{~kg} \mathrm{ha}^{-1}$ da formulação 2-20-20 (N-P $\left.\mathrm{O}_{5}-\mathrm{K}_{2} \mathrm{O}\right)$. Em dezembro de 2009 foi realizada a semeadura do milho, híbrido Impacto da empresa Syngenta, em profundidade de $3 \mathrm{~cm} \mathrm{e}$ espaçamento de $90 \mathrm{~cm}$ entrelinhas. A adubação de semeadura consistiu da aplicação de $300 \mathrm{~kg} \mathrm{ha}^{-1}$ da formulação N-P-K (820-20); a adubação de cobertura foi realizada no estádio V6 da cultura com $250 \mathrm{~kg} \mathrm{ha}^{-1}$ da formulação N- $\mathrm{P}_{2} \mathrm{O}_{5}-\mathrm{K}_{2} \mathrm{O}$ (20-0-10) em superfície, ao lado da linha de plantio. As análises químicas foram realizadas conforme Raij et al. (2001) (Tabela 1).

Os tratamentos para o ano agrícola de 2008/09 foram: SPD5, SPD7, SPD9, SPC e uma área adjacente de mata nativa como controle $(\mathrm{MN})$. Os tratamentos se estenderam para o ano agrícola de 2009/10, assim identificados: SPD6, SPD8, SPD10, SPC e MN. Os experimentos foram conduzidos em delineamento inteiramente casualizado e em parcelas subdivididas, com quatro repetições. As parcelas foram constituídas pelos tratamentos e as subparcelas pelas camadas de solo amostradas $(0-10 ; 10-20$ e $20-30 \mathrm{~cm})$.

Para avaliar os atributos físicos do solo durante o florescimento das culturas amostras indeformadas foram coletadas utilizando-se cilindros de 53,16 $10^{-6} \mathrm{~m}^{3}$, para determinar a densidade do solo (Ds), a macroporosidade e a microporosidade do solo por secagem em mesa de tensão $(0,006$ MPa) (EMBRAPA, 2011). Visando à determinação do teor de matéria orgânica (MO) coletaram-se amostras de solo com estrutura deformada, por meio de trado holandês, realizandose cinco amostragens simples para compor uma amostra composta por repetição. As amostras foram acondicionadas em caixas identificadas, secas ao ar e encaminhadas ao laboratório onde foram destorroadas e submetidas à análise conforme descrito por Raij et al. (2001). O teor de MO foi convertido

Tabela 1. Características químicas do Latossolo Vermelho na camada de $0-20 \mathrm{~cm}$, antes da adubação

\begin{tabular}{|c|c|c|c|c|c|c|c|}
\hline \multirow{2}{*}{$\begin{array}{c}\mathrm{pH}\left(\mathrm{CaCl}_{2}\right) \\
1: 2,5\end{array}$} & \multirow{2}{*}{$\begin{array}{c}\mathbf{P} \\
\left.(\mathrm{mg} \mathrm{dm})^{-3}\right)\end{array}$} & $\bar{K}$ & $\mathrm{Ca}$ & $\overline{M g}$ & SB & $\overline{\text { CTC }}$ & \multirow{2}{*}{$\begin{array}{c}V \\
(\%)\end{array}$} \\
\hline & & \multicolumn{5}{|c|}{$\left(\mathrm{mmol}_{\mathrm{c}} \mathrm{dm}^{-3}\right)$} & \\
\hline \multicolumn{8}{|c|}{ 2008/09 } \\
\hline 5,5 & 40 & 2,4 & 28 & 17 & 46 & 71 & 64 \\
\hline \multicolumn{8}{|c|}{$2009 / 10$} \\
\hline 5,2 & 39 & 1,1 & 24 & 12 & 37 & 66 & 56 \\
\hline
\end{tabular}


em carbono orgânico total (COT) pela aplicação do fator de van Bemmelen $(1,724)$, com base no pressuposto de que a $\mathrm{MO}$ possui $58 \%$ de CO (Fageria et al., 1999); portanto, o valor de MO foi dividido por 1,724 .

Com a determinação da Ds e do teor de C total do solo foi possível calcular o estoque de carbono do solo pelo método da camada (Bayer et al., 2000) e da massa de solo equivalentes (Ellert \& Bettany, 1995). O método da camada equivalente leva em consideração a espessura da camada e a densidade do solo enquanto o método da massa equivalente de solo utiliza, como referência, a massa de solo de um tratamento a qual é tomada como base para o cálculo do estoque nos demais tratamentos. Para este estudo foram consideradas como referência as massas de solo das camadas correspondentes à mata nativa a qual representa a condição original do solo. O cálculo dos estoques para a camada equivalente foi realizado pela Eq. 1:

$$
\mathrm{EC}=\frac{(\mathrm{Ds} \times \mathrm{h} \times \mathrm{COT})}{10}
$$

onde:
EC - estoque de carbono, $\mathrm{t} \mathrm{ha}^{-1}$
Ds - densidade do solo, $\mathrm{kg} \mathrm{dm}^{-3}$
$\mathrm{H}$ - espessura da camada amostrada, $\mathrm{cm}$
COT - teor de C orgânico total, $\mathrm{g} \mathrm{kg}^{-1}$

Os resultados foram submetidos à análise de variância com aplicação do teste $\mathrm{F}$ e os valores médios comparados entre si pelo teste de Tukey $(\mathrm{p}<0,05)$, com auxílio do programa Assistat.

\section{Resultados E Discussão}

O teor de carbono orgânico total (COT) na MN foi superior na camada de $0-10 \mathrm{~cm}(2008 / 09$ e $2009 / 10)$ e somente na camada de 10-20 cm na última safra (2009/10) em relação aos demais tratamentos (Tabela 2). Este comportamento pode estar associado à deposição contínua de serapilheira além da referida área apresentar ausência de interferência antrópica (Guareschi et al., 2012). Schiavo et al. (2011) também verificaram maiores teores de COT em Latossolo Vermelho sob vegetação de cerrado nas camadas de 0-5; 5-10 e 10-30 cm em comparação aos manejos de plantio direto com integração lavoura-pecuária sob os usos de milho, braquiária e capimtanzânia. Neste aspecto se observa que os teores de COT nos tratamentos SPD5, SPD7, SPD6 e SPD8 na camada de 0-10 cm não apresentaram diferença em relação ao SPC. Uma situação próxima a esta também foi constatada por Calonego et al. (2011) em Argissolo Vermelho em SPD por quatro anos; é provável que esta manifestação tenha ocorrido por se tratar dos primeiros anos de implantação do sistema; outra justificativa é que o local do presente estudo é caracterizado por baixos índices pluviométricos no outono-inverno e alta temperatura e umidade durante o verão oferecendo, portanto, condições inadequadas para manter a palhada sobre o solo e favorece a rápida decomposição da $\mathrm{MO}$.

Os tratamentos com maiores anos de implantação dos SPD na camada superficial do solo aumentaram os aportes de COT (SDP9 13,22 $\mathrm{g} \mathrm{kg}^{-1}$ e no SPD10 11,99 $\mathrm{g} \mathrm{kg}^{-1}$,
Tabela 2. Teores de carbono orgânico total (COT) e densidade (Ds) de um Latossolo Vermelho sob diferentes tempos de adoção de manejos e mata nativa em camadas

\begin{tabular}{|c|c|c|c|c|c|}
\hline \multirow[t]{2}{*}{ Tratamento } & $\begin{array}{c}\text { COT } \\
\left(g_{k^{-1}}\right)\end{array}$ & $\begin{array}{c}\text { Ds } \\
\left(\mathrm{kg} \mathrm{dm}^{-3}\right)\end{array}$ & \multirow[t]{2}{*}{ Tratamento } & $\begin{array}{c}\text { COT } \\
\left(g_{\mathrm{kg}^{-1}}\right)\end{array}$ & $\begin{array}{c}\text { Ds } \\
\left(\mathrm{kg} \mathrm{dm}^{-3}\right)\end{array}$ \\
\hline & \multicolumn{2}{|c|}{$2008 / 09$} & & \multicolumn{2}{|c|}{$2009 / 10$} \\
\hline \multicolumn{6}{|c|}{$0-10 \mathrm{~cm}$} \\
\hline MN & $16,42 \mathrm{Aa}$ & $1,39 \mathrm{Bb}$ & MN & $15,04 \mathrm{Aa}$ & $1,27 \mathrm{Cb}$ \\
\hline SPD5 & $9,15 \mathrm{Ca}$ & $1,68 \mathrm{Aa}$ & SPD6 & $9,23 \mathrm{Ca}$ & $1,65 \mathrm{Ab}$ \\
\hline SPD7 & $9,88 \mathrm{Ca}$ & $1,63 \mathrm{Aa}$ & SPD8 & $9,23 \mathrm{Ca}$ & $1,79 \mathrm{Aa}$ \\
\hline SPD9 & $13,22 \mathrm{Ba}$ & 1,58 $\mathrm{Aa}$ & SPD10 & $11,99 \mathrm{Ba}$ & 1,69 Aa \\
\hline SPC & $9,44 \mathrm{Ca}$ & $1,43 \mathrm{Bb}$ & SPC & $8,07 \mathrm{Ca}$ & $1,47 \mathrm{Bb}$ \\
\hline \multicolumn{6}{|c|}{$10-20 \mathrm{~cm}$} \\
\hline MN & $10,75 \mathrm{Ab}$ & 1,54 Ba & MN & $10,76 \mathrm{Ab}$ & $1,62 \mathrm{Aa}$ \\
\hline SPD5 & 6,83 Cb & $1,58 \mathrm{ABb}$ & SPD6 & 7,05 Bb & $1,76 \mathrm{Aa}$ \\
\hline SPD7 & $8,86 \mathrm{ABa}$ & $1,67 \mathrm{Aa}$ & SPD8 & $6,76 \mathrm{Bb}$ & $1,75 \mathrm{Aa}$ \\
\hline SPD9 & $9,59 \mathrm{ABb}$ & $1,57 \mathrm{ABa}$ & SPD10 & $7,12 \mathrm{Bb}$ & $1,76 \mathrm{Aa}$ \\
\hline SPC & $8,43 \mathrm{BCa}$ & $1,52 \mathrm{Ba}$ & SPC & $7,12 \mathrm{Bb}$ & 1,69 Aa \\
\hline \multicolumn{6}{|c|}{$20-30 \mathrm{~cm}$} \\
\hline MN & $9,74 A b$ & $1,56 \mathrm{BCa}$ & MN & $7,92 \mathrm{AC}$ & $1,57 \mathrm{Ba}$ \\
\hline SPD5 & $6,25 \mathrm{Cb}$ & $1,60 \mathrm{ABab}$ & SPD6 & 7,27 Ab & 1,71 ABab \\
\hline SPD7 & $7,27 \mathrm{BCb}$ & 1,69 Aa & SPD8 & $5,23 \mathrm{BC}$ & $1,78 \mathrm{Aa}$ \\
\hline SPD9 & $9,16 \mathrm{ABb}$ & $1,60 \mathrm{ABa}$ & SPD10 & 7,92 Ac & $1,74 \mathrm{ABa}$ \\
\hline SPC & $6,68 \mathrm{Cb}$ & 1,49 Cab & SPC & $6,61 \mathrm{ABb}$ & $1,71 \mathrm{ABa}$ \\
\hline CV $(\%)^{1}$ & 11,58 & 4,08 & & 15,84 & 6,97 \\
\hline CV $(\%)^{2}$ & 9,71 & 3,17 & & 5,86 & 3,67 \\
\hline
\end{tabular}

Médias seguidas da mesma letra não diferem entre si pelo teste de Tukey $(p<0,05)$. Letras maiúsculas referem-se à comparação de tratamentos em uma mesma camada em dado ano e as minúsculas comparam médias de camadas do tratamento em determinado ano. ${ }^{1}$ Coeficiente de variação referente aos tratamentos (parcelas); ${ }^{2}$ Coeficiente de variação referente às camadas (subparcelas). Ano agrícola de 2008/09: MN: mata nativa; SPD5: plantio direto com 5 anos; SPD7: plantio direto com 7 anos; SPD9: plantio direto com 9 anos e SPC: preparo convencional. Ano agrícola de 2009/10: MN: mata nativa; SPD6: plantio direto com 6 anos; SPD8: plantio direto com 8 anos; SPD10: plantio direto com 10 anos; e SPC: preparo convencional no Latossolo Vermelho

respectivamente, em 2008/09 e 2009/10) quando comparados aos demais tratamentos em SPD e SPC. No caso do preparo convencional o próprio preparo do solo com gradagem e escarificação incorpora os resíduos vegetais em profundidade e contribui para a oxidação da matéria orgânica do solo. Pode-se considerar que nas camadas subsuperficiais a longa duração de uso contínuo da área como sistema conservacionista (SPD10) foi eficiente em aumentar o teor de COT em relação aos SPD5 e SPD8, com exceção deste último na camada de 10-20 cm. Resultado oposto foi relatado por Leite et al. (2010) em plantio direto durante seis anos, em Latossolo do cerrado piauiense.

Neste estudo e em todas as camadas avaliadas, constatou-se pelo menos um tratamento em SPD com teor semelhante de COT ao SPC. Ressalta-se que em 2009/10 o SPD10 apresentou, na camada de $20-30 \mathrm{~cm}$, teor de COT semelhante ao SPC porém o sistema conservacionista tem aproximadamente $20 \%$ a mais de COT do que no preparo convencional cuja justificativa se restringe ao ano de implantação dos SPD em comparação com o estudo de Hickmann \& Costa (2012) os quais constataram que, realmente, o manejo de plantio direto com 23 anos aumentou o teor de carbono orgânico do solo em referência ao preparo convencional; por sua vez, os menores valores de carbono orgânico nos sistemas conservacionistas, além do menor tempo de implantação, também podem ser atribuído à textura média que favorece a decomposição da matéria orgânica e não exclusivamente à deposição de resíduos vegetais.

Os teores de COT do sistema plantio direto (2008/09) na camada de $0-10 \mathrm{~cm}$ foram inferiores em comparação com a MN. Verificou-se, nesta camada do SPD, que quando conduzidos no ano de 2009/10, os teores de COT foram 
mantidos em relação ao ano de 2008/09; desta forma, o tempo de implantação do SPD (5 a 10 anos) ainda não foi suficiente para se equiparar aos teores de COT da $\mathrm{MN}$; entretanto se verificaram 40 e $48,7 \%$ a mais de COT na camada superficial, respectivamente nos SPD9 e SPD10 que no SPC. Como se pode observar, a transição desses anos agrícolas promoveu um incremento de aproximadamente $9 \%$ de COT ressaltando como é fundamental considerar o tempo de duração do sistema conservacionista; reafirmando este comportamento, Costa Jr. et al. (2012) verificaram uma contribuição mais expressiva para o acúmulo sob sistema plantio direto de 15 anos na camada de 0-5 cm em Latossolo Vermelho em comparação ao plantio convencional e pastagem. Segundo referido no contexto desses autores, tal fato é devido, possivelmente, ao maior contato com os resíduos culturais deixados na superfície que são fontes de matéria orgânica no solo.

A densidade do solo na camada $0-10 \mathrm{~cm}$ do SPC foi significativamente menor em relação ao sistema plantio direto nos dois anos agrícolas avaliados (Tabela 3). De acordo com Silva et al. (2005), maiores valores de densidade do solo na superfície do sistema plantio direto em relação ao SPC, são verificados em muitos casos e são devidos ao adensamento natural em função da ausência de revolvimento e também pela compactação ocasionada pelo tráfego de máquinas. Fato semelhante ainda foi observado por Tavares Filho et al. (2012), em manejo de semeadura direta por onze anos no qual a densidade e a microporosidade do Nitossolo Vermelho foram 4,2 e 40,8\%, respectivamente, superiores em relação ao manejo de semeadura direta com escarificação quadrianual.

Tabela 3. Macroporosidade (Mac) e microporosidade (Mic) do Latossolo Vermelho sob diferentes tempos de adoção de manejos e mata nativa em camadas

\begin{tabular}{|c|c|c|c|c|c|}
\hline \multirow{3}{*}{ Tratamento } & Mac & Mic & \multirow{3}{*}{ Tratamento } & Mac & Mic \\
\hline & \multirow{2}{*}{\multicolumn{2}{|c|}{$\frac{\left(m^{3}\right.}{2008 / 09} \frac{\left.m^{-3}\right)}{108}$}} & & \multicolumn{2}{|c|}{$\left(m^{3} m^{-3}\right)$} \\
\hline & & & & \multicolumn{2}{|c|}{$2009 / 10$} \\
\hline \multicolumn{6}{|c|}{$0-10 \mathrm{~cm}$} \\
\hline MN & $0,196 \mathrm{Aa}$ & $0,243 \mathrm{Ca}$ & MN & $0,275 \mathrm{Aa}$ & $0,263 \mathrm{Cb}$ \\
\hline SPD5 & $0,096 \mathrm{Ba}$ & $0,276 \mathrm{ABa}$ & SPD6 & $0,141 \mathrm{Aa}$ & $0,312 \mathrm{Ba}$ \\
\hline SPD7 & $0,133 \mathrm{Ba}$ & $0,239 \mathrm{Ca}$ & SPD8 & $0,102 \mathrm{Aa}$ & $0,262 \mathrm{Cb}$ \\
\hline SPD9 & $0,106 \mathrm{Ba}$ & $0,296 \mathrm{Ab}$ & SPD10 & 0,098 Aa & $0,347 \mathrm{Aa}$ \\
\hline SPC & $0,213 \mathrm{Aa}$ & $0,257 \mathrm{BCa}$ & SPC & $0,245 \mathrm{Aa}$ & $0,262 \mathrm{Cb}$ \\
\hline \multicolumn{6}{|c|}{$10-20 \mathrm{~cm}$} \\
\hline MN & $0,150 \mathrm{Ab}$ & $0,252 \mathrm{Ba}$ & MN & $0,145 \mathrm{Aa}$ & $0,306 \mathrm{ABa}$ \\
\hline SPD5 & $0,121 \mathrm{ABa}$ & $0,260 \mathrm{Ba}$ & SPD6 & $0,274 \mathrm{Aa}$ & $0,302 \mathrm{ABa}$ \\
\hline SPD7 & $0,111 \mathrm{ABa}$ & $0,235 \mathrm{Ba}$ & SPD8 & $0,141 \mathrm{Aa}$ & $0,290 \mathrm{Ba}$ \\
\hline SPD9 & $0,098 \mathrm{Ba}$ & 0,299 Aab & SPD10 & $0,189 \mathrm{Aa}$ & $0,331 \mathrm{Aa}$ \\
\hline SPC & $150 \mathrm{Ab}$ & $0,257 \mathrm{Ba}$ & SPC & $0,148 \mathrm{Aa}$ & $0,290 \mathrm{Ba}$ \\
\hline \multicolumn{6}{|c|}{$20-30 \mathrm{~cm}$} \\
\hline MN & $0,125 \mathrm{ABb}$ & $0,257 \mathrm{BCa}$ & MN & $0,180 \mathrm{Aa}$ & $0,297 \mathrm{Ba}$ \\
\hline SPD5 & $0,111 \mathrm{BCa}$ & $0,268 \mathrm{Ba}$ & SPD6 & $0,131 \mathrm{Aa}$ & $0,298 \mathrm{Ba}$ \\
\hline SPD7 & $0,109 \mathrm{BCa}$ & $0,228 \mathrm{Ca}$ & SPD8 & $0,138 \mathrm{Aa}$ & $0,299 \mathrm{ABa}$ \\
\hline SPD9 & $0,067 \mathrm{Ca}$ & $0,316 \mathrm{Aa}$ & SPD10 & $0,091 \mathrm{Aa}$ & $0,329 \mathrm{Aa}$ \\
\hline SPC & $0,171 \mathrm{Aab}$ & $0,249 \mathrm{BCa}$ & SPC & $0,146 \mathrm{Aa}$ & $0,299 \mathrm{ABa}$ \\
\hline CV (\%) ${ }^{1}$ & 16,19 & 8,18 & & 22,97 & 5,97 \\
\hline CV $(\%)^{2}$ & 18,94 & 3,91 & & 21,05 & 4,50 \\
\hline
\end{tabular}

Médias seguidas da mesma letra não diferem entre si pelo teste de Tukey $(p<0,05)$. Letras maiúsculas referem-se à comparação de tratamentos em uma mesma camada em dado ano e as minúsculas comparam médias de camadas do tratamento em determinado ano. ${ }^{1}$ Coeficiente de variação referente aos tratamentos (parcelas); ${ }^{2}$ Coeficiente de variação referente às camadas (subparcelas). Ano agrícola de 2008/09: MN: mata nativa; SPD5: plantio direto com 5 anos; SPD7: plantio direto com 7 anos; SPD9: plantio direto com 9 anos e SPC: preparo convencional. Ano agrícola de 2009/10: MN: mata nativa; SPD6: plantio direto com 6 anos; SPD8: plantio direto com 8 anos; SPD10: plantio direto com 10 anos e SPC: preparo convencional no Latossolo Vermelho
A macroporosidade do solo na camada $0-10 \mathrm{~cm}$ do SPD5 $\left(0,096 \mathrm{~m}^{3} \mathrm{~m}^{-3}\right)$, nas camadas $10-20 \mathrm{~cm}$ e $20-30 \mathrm{~cm}$ do SPD9 $\left(0,098\right.$ e $0,067 \mathrm{~m}^{3} \mathrm{~m}^{-3}$, respectivamente $)$ no ano de 2008/09, foi próxima a $0,10 \mathrm{~m}^{3} \mathrm{~m}^{-3}$ (Tabela 3 ), mínimo adequado para as trocas líquidas e gasosas entre o ambiente externo e o solo considerado crítico para o crescimento das raízes da maioria das culturas (Baver et al., 1972). Estão também próximos desse volume crítico de macroporosidade o SPD10 nas camadas de $0-10 \mathrm{~cm}\left(0,098 \mathrm{~m}^{3} \mathrm{~m}^{-3}\right)$ e de $0,20-0,30 \mathrm{~m}\left(0,091 \mathrm{~m}^{3} \mathrm{~m}^{-3}\right)$ no ano de 2009/10. Assim, quando o tratamento SPD9 passou a ser SPD10 no ano de 2009/10 observou-se, na camada de 20-30 $\mathrm{cm}$, um acréscimo de macroporosidade do solo.

De acordo com Silva et al. (2005), normalmente o revolvimento do solo promove um aumento temporário da macroporosidade quando comparado ao sistema plantio direto. Esses mesmos autores afirmaram que mencionado efeito é, entretanto, eliminado pela reconsolidação ou adensamento natural do solo. Maiores valores de microporosidade foram verificados no solo sob SPD5 e SPD9 a 0-10 cm de profundidade e no SPD9 na camada de 10-30 cm em comparação com os SPD (ano de 2008/09) visto que, segundo Reichert et al. (2007) durante o processo de adensamento do solo os macroporos responsáveis pela aeração do solo diminuem e são substituídos por microporos; apenas o menor volume de microporos no ano 2008/09 no SPD7 na camada de 20-30 $\mathrm{cm}$, quando passou a ser SPD8 no ano de 2009/10 teve um acréscimo da microporosidade do solo. Toda a profundidade de solo avaliada neste estudo, ou seja, a camada de $0-30 \mathrm{~cm}$, foi fundamental em avaliar a susceptibilidade da densidade, macro e microporosidade do solo principalmente quando se considera que em toda esta camada foram diagnosticados valores críticos de macroporosidade próximos a $0,10 \mathrm{~m}^{3} \mathrm{~m}^{-3}$.

Em relação aos estoques de carbono (EC), os métodos de cálculo de camada equivalente (Bayer et al., 2000) e de massa equivalente (Ellert \& Bettany, 1995) resultaram em valores muito similares (Tabelas 4 e 5), graças à pequena variação na Ds entre os tratamentos (Tabela 2). Por ser teoricamente o método mais correto, a discussão dos efeitos dos tratamentos nos EC foi baseada nos resultados obtidos pelo método da massa equivalente de solo.

Os tratamentos SPD9 (2008/09) e SPD10 (2009/10) apresentaram os maiores EC, em comparação aos demais SPD na camada de $0-10 \mathrm{~cm}$ (Tabelas 4 e 5). A diferença entre tratamentos seguiu a ordem decrescente $\mathrm{MN}>$ SPD9 > SPD5 = SPD7 $=$ SPC em 2008/09 e MN > SPD10 > SPD8 = SPD6 = SPC em 2009/10. O maior tempo de implantação em SPD tem efeito com a deposição de resíduos culturais associado ao florescimento (período seco) de todos os anos mas a ausência de revolvimento pode ter justificado uma parte deste resultado. Campos et al. (2013) relataram que o plantio direto por nove anos em Latossolo Amarelo distrófico acumulou mais $\mathrm{C}$ no período seco, com 44,9 e $26,75 \mathrm{Mg} \mathrm{ha}^{-1}$ para as camadas de 0-20 e 20-40 cm, respectivamente em comparação ao período chuvoso. Considera-se que a similaridade no estoque de $\mathrm{C}$ entre as áreas de cerrado natural e sistema plantio direto nas camadas superficiais do solo decorre do maior aporte de resíduos vegetais depositados na superfície do solo (Gazolla et al., 2013). Corroborando esses resultados, Guareschi et al. (2012) também constataram, ao avaliar uma cronossequência 
Tabela 4. Estoque de carbono orgânico calculado em camada equivalente de solo e em massa equivalente de Latossolo Vermelho referente a 2008/09, em diferentes tempos de adoção de manejos e mata nativa em camadas

\begin{tabular}{|c|c|c|c|c|}
\hline \multirow{3}{*}{ Tratamento } & \multicolumn{2}{|c|}{ Camada } & \multicolumn{2}{|c|}{ Massa } \\
\hline & \multicolumn{4}{|c|}{ Equivalente de solo } \\
\hline & Massa & Carbono & Massa ${ }^{(2)}$ & Carbono \\
\hline & \multicolumn{4}{|c|}{$\left(\mathrm{t} \mathrm{ha}^{-1}\right)$} \\
\hline & \multicolumn{4}{|c|}{$0-10 \mathrm{~cm}^{(1)}$} \\
\hline MN & 739 & $22,78 \mathrm{Aa}$ & 739 & $22,78 \mathrm{Aa}$ \\
\hline SPD5 & 893 & $15,37 \mathrm{Ba}$ & 739 & $12,72 \mathrm{Ca}$ \\
\hline SPD7 & 866 & $16,16 \mathrm{Ba}$ & 739 & $13,80 \mathrm{Ca}$ \\
\hline SPD9 & 840 & $20,89 \mathrm{Aa}$ & 739 & $18,38 \mathrm{Ba}$ \\
\hline \multirow[t]{2}{*}{ SPC } & 760 & $13,50 \mathrm{Ba}$ & 739 & $13,17 \mathrm{Ca}$ \\
\hline & \multicolumn{4}{|c|}{$10-20 \mathrm{~cm}^{(1)}$} \\
\hline MN & 819 & $16,49 \mathrm{Ab}$ & 819 & $16,49 \mathrm{Ab}$ \\
\hline SPD5 & 840 & $10,79 \mathrm{Cb}$ & 819 & $10,52 \mathrm{Cab}$ \\
\hline SPD7 & 888 & $14,77 \mathrm{ABa}$ & 819 & $13,62 \mathrm{ABa}$ \\
\hline SPD9 & 835 & $15,04 \mathrm{ABb}$ & 819 & $14,75 \mathrm{ABb}$ \\
\hline \multirow[t]{2}{*}{ SPC } & 808 & $12,80 \mathrm{BCa}$ & 819 & $12,97 \mathrm{BCa}$ \\
\hline & \multicolumn{4}{|c|}{$20-30 \mathrm{~cm}^{(1)}$} \\
\hline MN & 829 & $15,25 \mathrm{Ab}$ & 829 & $15,25 \mathrm{Ab}$ \\
\hline SPD5 & 850 & $10,00 \mathrm{Cb}$ & 829 & $9,76 \mathrm{Bb}$ \\
\hline SPD7 & 898 & $12,22 \mathrm{BCb}$ & 829 & $11,29 \mathrm{Bb}$ \\
\hline SPD9 & 850 & $14,62 \mathrm{ABb}$ & 829 & $14,25 \mathrm{Ab}$ \\
\hline SPC & 792 & $9,93 \mathrm{Cb}$ & 829 & $10,40 \mathrm{Bb}$ \\
\hline CV $(\%)^{3}$ & & 11,20 & & 11,54 \\
\hline CV $(\%)^{4}$ & & 9,57 & & 9,55 \\
\hline
\end{tabular}

${ }^{1}$ Limites teóricos de cada camada que contém a mesma massa de solo da mata nativa (referência). ${ }^{2}$ Massa de solo da mata nativa (referência), de acordo com os valores de densidade apresentados. Médias seguidas da mesma letra não diferem entre si pelo teste de Tukey $(p<0,05)$. Letras maiúsculas referem-se à comparação de tratamentos em uma mesma camada em dado ano e as minúsculas comparam médias de camadas do tratamento em determinado ano. ${ }^{3}$ Coeficiente de variação referente aos tratamentos (parcelas): ${ }^{4}$ Coeficiente de variação referente às camadas (subparcelas). Ano agrícola de 2008/09: MN: mata nativa; SPD5: plantio direto com 5 anos; SPD7: plantio direto com 7 anos; SPD9: plantio direto com 9 anos e SPC: preparo convencional.

de áreas com 15 e 20 anos de SPD em um Latossolo Vermelho no estado de Góias, semelhanças no estoque de $\mathrm{C}$ com o cerrado nativo nas camadas superficiais do solo.

$\mathrm{O}$ estoque de carbono no SPD10 foi superior em relação aos demais SPD somente na camada de $0-10 \mathrm{~cm}$ já que na 10-20 cm todos os SPD foram semelhantes, inclusive o SPC (2009/10) (Tabela 5). Conceição et al. (2013) observaram alto conteúdo e estoques de carbono no PD que no PC na camada de $0-5 \mathrm{~cm}$ em Argissolo de textura franco-argilo-arenosa após 18 anos de cultivo, fato também observado neste estudo porém para a camada superficial de $0-10 \mathrm{~cm}$. Os autores afirmam que tal comportamento em solos subtropicais depende do estabelecimento da diversidade de culturas empregadas na cobertura do solo ao invés da simples conversão do PC para PD. De acordo com Siqueira Neto et al. (2010) os estoques de $\mathrm{C} \mathrm{em}$ Latossolo Vermelho-Amarelo oscilaram entre 4,2 a $6,7 \mathrm{~kg} \mathrm{~m}^{-2}$, sendo os maiores também em condições antrópicas, como no cerrado e no plantio direto, durante 12 anos (PD 12) enquanto os valores mais baixos $\mathrm{s}$ foram encontrados sob pasto, preparo convencional e com um e quatro anos de plantio direto jovem ( $<4$ anos). Depois da adoção de 12 anos de plantio direto os estoques de $\mathrm{C}$ retornaram para os níveis daqueles sob vegetação natural, com nenhuma diferença significativa entre os estoques de C entre cerrado e PD12 (Siqueira Neto et al., 2010). Liu et al. (2013) observaram que o plantio direto na camada de 0-10 $\mathrm{cm}$ teve estoque de $\mathrm{C}$ superior ao do preparo convencional em Cambissolo crômico. Esses autores observaram que após 17 anos o PD apresentou um estoque acumulativo superior em
Tabela 5. Estoque de carbono orgânico calculado em camada equivalente de solo e em massa equivalente de Latossolo Vermelho, referente a 2009/10, em diferentes tempos de adoção de manejos e mata nativa em camadas

\begin{tabular}{|c|c|c|c|c|}
\hline \multirow{4}{*}{ Tratamento } & \multicolumn{2}{|c|}{ Camada } & \multicolumn{2}{|c|}{ Massa } \\
\hline & \multicolumn{4}{|c|}{ Equivalente de solo } \\
\hline & Massa & Carbono & Massa $^{(2)}$ & Carbono \\
\hline & \multicolumn{4}{|c|}{$\left(t h^{-1}\right)$} \\
\hline & \multicolumn{4}{|c|}{$0-10 \mathrm{~cm}^{(1)}$} \\
\hline MN & 675 & $19,22 \mathrm{ABa}$ & 675 & $19,22 \mathrm{Aa}$ \\
\hline SPD6 & 877 & $15,27 \mathrm{CDa}$ & 675 & $11,76 \mathrm{Ca}$ \\
\hline SPD8 & 951 & $16,53 \mathrm{BCa}$ & 675 & $11,73 \mathrm{Ca}$ \\
\hline SPD10 & 898 & $20,26 \mathrm{Aa}$ & 675 & $15,23 \mathrm{Ba}$ \\
\hline \multirow[t]{2}{*}{ SPC } & 781 & $12,71 \mathrm{Da}$ & 675 & $10,98 \mathrm{Ca}$ \\
\hline & \multicolumn{4}{|c|}{$10-20 \mathrm{~cm}^{(1)}$} \\
\hline MN & 861 & $17,41 \mathrm{Ab}$ & 861 & $17,41 \mathrm{Ab}$ \\
\hline SPD6 & 936 & $12,43 \mathrm{Bb}$ & 861 & $11,44 \mathrm{Ba}$ \\
\hline SPD8 & 930 & $11,34 \mathrm{Bb}$ & 861 & $10,50 \mathrm{Ba}$ \\
\hline SPD10 & 936 & $12,55 \mathrm{Bb}$ & 861 & $11,54 \mathrm{Bb}$ \\
\hline \multirow[t]{2}{*}{ SPC } & 898 & $12,02 \mathrm{Ba}$ & 861 & $11,52 \mathrm{Ba}$ \\
\hline & \multicolumn{4}{|c|}{$20-30 \mathrm{~cm}^{(1)}$} \\
\hline MN & 835 & $12,42 \mathrm{AC}$ & 835 & $12,42 \mathrm{Ac}$ \\
\hline SPD6 & 909 & $12,46 \mathrm{Ab}$ & 835 & $11,44 \mathrm{Aa}$ \\
\hline SPD8 & 946 & $9,35 \mathrm{Bc}$ & 835 & $8,24 \mathrm{Bb}$ \\
\hline SPD10 & 925 & $11,27 \mathrm{ABb}$ & 835 & $10,17 \mathrm{ABb}$ \\
\hline SPC & 909 & $12,42 \mathrm{Ac}$ & 835 & $10,35 \mathrm{ABa}$ \\
\hline CV $(\%)^{3}$ & & 15,80 & & 17,42 \\
\hline CV $(\%)^{4}$ & & 7,22 & & 7,41 \\
\hline
\end{tabular}

${ }^{1}$ Limites teóricos de cada camada que contém a mesma massa de solo da mata nativa (referência). ${ }^{2}$ Massa de solo da mata nativa (referência), de acordo com os valores de densidade apresentados. Médias seguidas da mesma letra não diferem entre si pelo teste de Tukey $(p<0,05)$. Letras maiúsculas referem-se à comparação de tratamentos em uma mesma camada em dado ano e as minúsculas comparam médias de camadas do tratamento em determinado ano. ${ }^{3}$ Coeficiente de variação referente aos tratamentos (parcelas); ${ }^{4}$ Coeficiente de variação referente às camadas (subparcelas). Ano agrícola de 2009/10: MN: mata nativa; SPD6: plantio direto com 6 anos; SPD8: plantio direto com 8 anos; SPD10: plantio direto com 10 anos; e SPC: preparo convencional

aproximadamente $8,3 \%\left(50,2 \mathrm{Mg} \mathrm{C}^{-1}\right)$ na camada superficial sendo que abaixo deste nível o estoque de $\mathrm{C}$ e a densidade do solo decresceram gradualmente com o aumento da profundidade atribuído aos baixos valores de resíduos vegetais.

Ressalta-se que em sistemas menos perturbados pelo preparo do solo, como o plantio direto (PD), após a decomposição dos resíduos vegetais, os estoques de carbono orgânico total (COT) podem aumentar na camada superficial. Neste sentido, Leite et al. (2010) constataram que o estoque de carbono na camada $0-20 \mathrm{~cm}$ em Latossolo Vermelho-Amarelo do cerrado piauiense foi maior no PD6, intermediário no PD2 com o PD4 não diferindo desses e menor no PC e na floresta nativa (FNC). Maiores valores de $\mathrm{COT}$ em Latossolo Amarelo no cerrado maranhense no PC em relação à $\mathrm{FNC}$, camada $0-40 \mathrm{~cm}$, podem ser atribuídos à baixa produção de biomassa das florestas de cerrado do meio-norte brasileiro (Azevedo et al., 2007). Além disso, a incorporação dos restos vegetais da cultura principal deixada sobre o solo possibilita a distribuição de $\mathrm{MO}$ por toda a camada arável permitindo que os teores de $\mathrm{CO}$ em profundidade maior possam ser semelhantes ou até maiores que nos sistemas conservacionistas (Ferreira et al., 2007). Este estudo se opõe à afirmação de existência de maiores estoques de C sob PD em comparação com os sistemas convencionais. Pode-se afirmar, que a maioria dos tratamentos manteve o teor de EC durante a transição da camada superficial para a subsuperficial contrariando, assim, o trabalho de Hickmann \& Costa (2012), os quais observaram decréscimo no EC total em profundidade em quatro tipos de manejo (convencional com preparos por grade de disco, arado de disco, arado de disco mais 
grade pesada, somente grade pesada e o plantio direto), por 23 anos de cultivo em Argissolo Vermelho-Amarelo; além disto, esses autores afirmaram que o menor estoque foi observado na camada mais profunda $(20-40 \mathrm{~cm})$ do solo embora apenas no arado e no gradeado.

\section{Conclusões}

1. Os anos de implantação de plantio direto de cinco a dez anos não foram eficientes em aumentar o conteúdo de carbono orgânico total na camada superficial.

2. O maior estoque de carbono foi mais pronunciado na camada superficial dentro da qual os sistemas plantio direto de nove e dez anos foram os mais relevantes em comparação com os demais sistemas conservacionistas.

3. Toda a profundidade de solo analisada foi fundamental para identificar a susceptibilidade dos atributos densidade, macro e microporosidade do solo.

\section{Agradecimentos}

À Fundação de Amparo à Pesquisa do Estado de São Paulo (FAPESP), pela concessão da bolsa de estudos.

\section{Literatura Citada}

Al-Kaisi, M. M.; Yin, X. H. Tillage and crops residue effects on soil carbon and carbon dioxide emission in corn-soybean rotations. Journal of Environmental Quality, v.34, p.437-445, 2005. http:// dx.doi.org/10.2134/jeq2005.0437

Azevedo, D. M. P. de.; Leite, L. F. C.; Teixeira Neto, M. L.; Dantas, J. S. Atributos físicos e químicos de um Latossolo Amarelo e distribuição do sistema radicular da soja sob diferentes sistemas de preparo no cerrado maranhense. Revista Ciência Agronômica, v.38, p.32-40, 2007.

Baver, L. D.; Gardner, W. H.; Gardner, W. R. Soil physics. 4. ed. New York: John Wiley and Sons, 1972. 529p.

Bayer, C.; Mielniczuk, J.; Amado, T. J. C.; Martin Neto, L.; Fernandes, S. A. Organic matter storage in a sandy loam Acrisol affected by tillage and cropping systems in Southern Brazil. Soil and Tillage Research, v.54, p.101-109, 2000. http://dx.doi.org/10.1016/S01671987(00)00090-8

Calonego, J. C.; Santos, C. H. dos; Tiritan, C. S.; Cunha Júnior, J. R. Estoques de carbono e propriedades físicas de solos submetidos a diferentes sistemas de manejo. Revista Caatinga, v.24, p.128135, 2011.

Campos, L. P.; Leite, L. F. C.; Maciel, G. A.; Brasil, E. L.; Iwata, B. F. Estoques e funções de carbono orgânico em Latossolo Amarelo submetido a diferentes sistemas de manejo. Pesquisa Agropecuária Brasileira, v.48, p.304-312, 2013. http://dx.doi.org/10.1590/S0100204X2013000300009

Cerri, C. C.; Bernoux, M.; Maia, S. M. F.; Cerri, C. E. P.; Costa Júnior, C.; Feigl, B. J.; Frazão, L. A.; Mello, F. F. C.; Galdos, M. V.; Moreira, C. S.; Carvalho, J. L. N. Greenhouse gas mitigation options in Brazil for land-use change, livestock and agriculture. Scientia Agricola, v.67, p.102-116, 2010. http://dx.doi.org/10.1590/S010390162010000100015

Conceição, P. C.; Dieckow, J.; Bayer, C. Combined role of no-tillage and cropping systems in soil carbon stocks and stabilization. Soil and Tillage Research, v.129, p.40-47, 2013. http://dx.doi. org/10.1016/j.still.2013.01.006
Costa Júnior, C.; Píccolo, M. C.; Siqueira Neto, M.; Camargo, P. B. de; Cerri, C. C.; Bernoux, M. Carbono em agregados do solo sob vegetação nativa, pastagem e sistemas agrícolas no bioma cerrado. Revista Brasileira de Ciência do Solo, v.36, p.1311-1321, 2012. http://dx.doi.org/10.1590/S0100-06832012000400025

EMBRAPA - Empresa Brasileira de Pesquisa Agropecuária Centro Nacional de Pesquisas de Solos. Manual de métodos de análises de solos. 2.ed. revista. Rio de Janeiro: Embrapa Solos, 2011. 230p. <http://www.cnps.embrapa.br/publicacoes/>. 3 Mar 2012.

EMBRAPA - Empresa Brasileira de Pesquisa Agropecuária. Sistema brasileiro de classificação de solos. 3.ed. Rio de Janeiro: Embrapa Solos, 2013. 353p.

Ellert, B. H.; Bettany, J. R. Calculation of organic matter and nutrients stored in soils under contrasting management regimes. Canadian Journal Soil Science, v.75, p.529-538, 1995. http://dx.doi.org/10.4141/ cjss95-075

Fageria, N. K.; Stone, F. F.; Santos, A. B. dos. Maximização da produção das culturas. Santo Antônio de Góias: Embrapa SCT/Embrapa CNPAF, 1999. 294p.

Ferreira, E. A. B.; Resck, D. V. S.; Gomes, A. C.; Ramos, M. L. G. Dinâmica do carbono da biomassa microbiana em cinco épocas do ano em diferentes sistemas de manejo do solo no cerrado. Revista Brasileira de Ciência do Solo, v.31, p.1625-1635, 2007. http://dx.doi.org/10.1590/S0100-06832007000600038

Franzluebbers, A. J.; Schomberg, H. H.; Endale, D. M. Surface-soil responses to paraplowing of long-term no-tillage cropland in the Southern Piedmont USA. Soil and Tillage Research, v.96, p.303315, 2007. http://dx.doi.org/10.1016/j.still.2007.07.001

Gazolla, P. R.; Guareschi, R. F.; Perin, A. Estoque de carbono e atributos físicos de um Latossolo Vermelho em diferentes sistemas de manejo. Revista Brasileira de Ciências Agrárias, v.8, p.229-235, 2013. http://dx.doi.org/10.5039/agraria.v8i2a2545

Guareschi, R. F.; Pereira, M. G.; Perin, A. Deposição de resíduos vegetais, matéria orgânica leve, estoques de carbono e nitrogênio e fósforo remanescente sob diferentes sistemas de manejo no cerrado goiano. Revista Brasileira de Ciência do Solo, v.36, p.909920, 2012. http://dx.doi.org/10.1590/S0100-06832012000300021

Hickmann, C.; Costa, L. M. da. Estoque de carbono no solo e agregados em Argissolo sob diferentes manejos de longa duração. Revista Brasileira de Engenharia Agrícola e Ambiental, v.16, p.1055-1061, 2012. http://dx.doi.org/10.1590/S1415-43662012001000004

Leite, L. F. C.; Galvão, S. R. S.; Holanda Neto, M. R.; Araújo, F. S.; Iwata, B. F. Atributos químicos e estoques de carbono em Latossolo sob plantio direto no cerrado do Piauí. Revista Brasileira de Engenharia Agrícola e Ambiental, v.14, p.1273-1280, 2010. http:// dx.doi.org/10.1590/S1415-43662010001200004

Liu, E.; Teclemariam, S. G.; Yan, C.; Yu, J.; Gu, R.; Liu, S.; He, W.; Liu, Q. Long-term effects of no-tillage management practice on soil organic carbon and its fractions in the northen China. Geoderma, v.213, p.379-384, 2013. http://dx.doi.org/10.1016/j. geoderma.2013.08.021

Raij, B. van.; Andrade, J. C. de; Cantarella, H.; Quaggio, J. A. Análise química para avaliação da fertilidade de solos tropicais. Campinas: Instituto Agronômico, 2001, 285p.

Reichert, J. M.; Suzuki, L. E. A. S.; Reinert, D. J. Compactação do solo em sistemas agropecuários e florestais: Identificação, efeitos, limites críticos e mitigação. In: Ceretta, C. A.; Silva, L. S.; Reichert, J. M. (ed.) Tópicos em ciência do solo. 5.ed. Viçosa: SBCS, 2007. p.49-134. 
Schiavo, J. A.; Rosset, J. S.; Pereira, M. G.; Salton, J. C. Índice de manejo de carbono e atributos químicos de Latossolo Vermelho sob diferentes sistemas de manejo. Pesquisa Agropecuária Brasileira, v.46, p.1332-1338, 2011. http://dx.doi.org/10.1590/ S0100-204X2011001000029

Silva, C. G. da.; Alves Sobrinho, T.; Vitorino, A. C. T.; Carvalho, D. F. de. Atributos físicos, químicos e erosão entressulcos sob chuva simulada, em sistemas de plantio direto e convencional. Engenharia Agrícola, v.25, p.144-153, 2005. http://dx.doi. org/10.1590/S0100-69162005000100016

Siqueira Neto, M.; Scopel, E.; Corbeels, M.; Cardoso, A. N.; Donzet, J-M.; Feller, C.; Piccolo, M. C.; Cerri, C. C.; Bernoux, M. Soil carbon stocks under no-tillage mulch-based cropping systems in the Brazilian Cerrado: An on-farm synchronic assessment. Soil and Tillage Research, v.110, p.187-195, 2010. http://dx.doi. org/10.1016/j.still.2010.07.010
Stone, L. F.; Silveira, P. M. Efeitos do sistema de preparo e da rotação de culturas na propriedade e densidade do solo. Revista Brasileira de Ciência do Solo, v.25, p.395-401, 2001. http://dx.doi.org/10.1590/ S0100-06832001000200015

Tavares Filho, J.; Guimarães, M. F.; Curmi, P.; Tessier, D. Physical properties of an Alfisol and no-till soybean yield. Revista Brasileira de Ciência do Solo, v.36, p.253-260, 2012. http://dx.doi. org/10.1590/S0100-06832012000100026

Tormena, C. A.; Roloff, G.; Sá, J. C. M. Propriedades físicas do solo sob plantio direto influenciadas por calagem, preparo inicial e tráfego. Revista Brasileira de Ciência do Solo, v.22, p.301-309, 1998. http:// dx.doi.org/10.1590/S0100-06831998000200016

Vezzani, F. M.; Mielniczuk, J. Agregação e estoque de carbono em Argissolo submetido a diferentes práticas de manejo agrícola, Revista Brasileira de Ciência do Solo, v.35, p.213-223, 2011. http:// dx.doi.org/10.1590/S0100-06832011000100020 